\title{
A note on quotients of strongly regular graphs
}

\author{
Michael Giudici *, Murray R. Smith \\ School of Mathematics and Statistics, The University of Western Australia \\ 35 Stirling Highway, WA 6009, Australia
}

Received 9 March 2010, accepted 9 September 2010, published online 21 October 2010

\begin{abstract}
We give examples of vertex-transitive strongly regular graphs with a normal quotient which is neither complete nor strongly regular.
\end{abstract}

Keywords: Strongly regular graph.

Math. Subj. Class.: 05E30

\section{Results}

A strongly regular graph is a graph that is not complete and for which each vertex has valency $k$ and there exist integers $\lambda, \mu$ such that each pair of adjacent vertices have $\lambda$ common neighbours and each pair of non-adjacent vertices have $\mu$ common neighbours. Such a graph is usually denoted by $\operatorname{srg}(v, k, \lambda, \mu)$ where $v$ is the number of vertices.

One common method for studying graphs is by taking quotients. Given a partition $\mathcal{B}$ of the vertex set of a graph $\Gamma$, the quotient graph is the graph whose vertices are the parts of the partition $\mathcal{B}$ and two parts $B_{1}$ and $B_{2}$ are joined by an edge if there exist $v \in B_{1}$ and $w \in B_{2}$ such that $v$ is adjacent to $w$ in the original graph $\Gamma$. When $\mathcal{B}$ is the set of orbits of a normal subgroup $N$ of some group $G$ of automorphisms of $\Gamma$ we denote the quotient by $\Gamma_{N}$ and refer to it as a normal quotient. It was shown in [5] that if $\Gamma$ is a strongly regular graph with a group of automorphisms $G$ which acts transitively on the vertex set and edge set of $\Gamma$ then for a nontrivial normal subgroup $N$ of $G$, the normal quotient $\Gamma_{N}$ is either a complete graph or a strongly regular graph. The purpose of this note is to show that edge-transitivity is indeed required.

In Example 1.1, we provide a vertex-transitive, edge-intransitive strongly regular graph $\Gamma$ where we take $G$ to be the full automorphism group and obtain a normal quotient which is neither strongly regular nor complete. The graph $\Gamma$ also has the following interesting properties:

\footnotetext{
*The first author is supported by an Australian Research Fellowship.

E-mail addresses: giudici@maths.uwa.edu.au (Michael Giudici), murray@murraysmith.id.au (Murray R. Smith)
} 
$0,1,1,1,1,1,1,1,1,1,1,1,1,1,1,0,0,0,0,0,0,0,0,0,0,0,0,0,0,0,0,0,0,0,0,0$ $1,0,1,1,1,1,0,0,0,0,0,0,0,0,0,1,1,1,1,1,1,1,1,1,0,0,0,0,0,0,0,0,0,0,0,0$ $1,1,0,1,0,0,1,1,0,0,0,0,0,0,0,1,1,0,0,0,0,0,0,0,1,1,1,1,1,1,1,0,0,0,0,0$ $1,1,1,0,0,0,0,0,1,1,0,0,0,0,0,0,0,1,1,0,0,0,0,0,1,1,0,0,0,0,0,1,1,1,1,1$ $1,1,0,0,0,1,0,0,0,0,1,1,0,0,0,0,0,0,0,1,1,0,0,0,1,0,1,1,1,0,0,1,1,1,0,0$ $1,1,0,0,1,0,0,0,0,0,0,0,1,1,0,0,0,0,0,0,0,1,1,0,0,1,1,0,0,1,1,1,0,0,1,1$ $1,0,1,0,0,0,0,0,1,0,1,0,0,0,1,0,0,1,0,1,0,1,1,0,0,0,0,1,1,1,0,1,0,0,1,0$ $1,0,1,0,0,0,0,0,0,0,1,1,1,0,0,1,0,0,1,0,0,1,0,1,0,0,1,0,0,1,0,0,1,1,1,0$ $1,0,0,1,0,0,1,0,0,0,0,0,1,0,1,0,1,0,0,1,1,1,0,0,0,0,1,0,0,0,1,0,1,1,0,1$ $1,0,0,1,0,0,0,0,0,0,1,0,1,1,0,1,0,0,1,1,0,0,0,1,0,0,0,1,1,0,1,1,0,0,0,1$ $1,0,0,0,1,0,1,1,0,1,0,0,0,0,0,0,0,1,0,0,1,0,1,1,1,0,0,0,0,1,1,0,1,0,0,1$ $1,0,0,0,1,0,0,1,0,0,0,0,0,1,1,0,1,1,1,0,0,1,0,0,0,1,0,1,1,0,0,0,1,0,0,1$ $1,0,0,0,0,1,0,1,1,1,0,0,0,0,0,0,1,0,0,0,1,0,1,1,0,1,0,1,1,0,0,0,0,1,1,0$ $1,0,0,0,0,1,0,0,0,1,0,1,0,0,1,1,1,1,0,1,0,0,0,0,1,0,0,0,0,1,1,0,0,1,1,0$ $1,0,0,0,0,0,1,0,1,0,0,1,0,1,0,1,0,0,1,0,1,0,1,1,1,1,1,0,0,0,0,1,0,0,0,0$ $0,1,1,0,0,0,0,1,0,1,0,0,0,1,1,0,0,0,0,1,1,0,1,0,0,0,1,1,0,0,0,0,1,0,1,1$ $0,1,1,0,0,0,0,0,1,0,0,1,1,1,0,0,0,1,0,0,1,0,0,1,0,0,0,1,0,1,1,1,1,0,0,0$ $0,1,0,1,0,0,1,0,0,0,1,1,0,1,0,0,1,0,0,0,0,0,1,1,0,0,1,0,1,0,0,0,0,1,1,1$ $0,1,0,1,0,0,0,1,0,1,0,1,0,0,1,0,0,0,0,0,1,1,1,0,0,0,0,0,1,1,1,1,0,1,0,0$ $0,1,0,0,1,0,1,0,1,1,0,0,0,1,0,1,0,0,0,0,0,1,0,1,0,1,0,0,1,1,0,0,1,1,0,0$ $0,1,0,0,1,0,0,0,1,0,1,0,1,0,1,1,1,0,1,0,0,0,0,0,1,0,0,0,1,1,0,0,0,0,1,1$ $0,1,0,0,0,1,1,1,1,0,0,1,0,0,0,0,0,0,1,1,0,0,0,1,1,0,0,1,0,0,1,0,0,0,1,1$ $0,1,0,0,0,1,1,0,0,0,1,0,1,0,1,1,0,1,1,0,0,0,0,0,0,1,0,1,0,0,1,0,1,1,0,0$ $0,1,0,0,0,0,0,1,0,1,1,0,1,0,1,0,1,1,0,1,0,1,0,0,1,1,1,0,0,0,0,1,0,0,0,0$ $0,0,1,1,1,0,0,0,0,0,1,0,0,1,1,0,0,0,0,0,1,1,0,1,0,1,0,1,0,0,1,0,0,1,1,0$ $0,0,1,1,0,1,0,0,0,0,0,1,1,0,1,0,0,0,0,1,0,0,1,1,1,0,0,0,1,1,0,0,1,0,0,1$ $0,0,1,0,1,1,0,1,1,0,0,0,0,0,1,1,0,1,0,0,0,0,0,1,0,0,0,0,1,0,1,1,0,1,0,1$ $0,0,1,0,1,0,1,0,0,1,0,1,1,0,0,1,1,0,0,0,0,1,1,0,1,0,0,0,0,0,0,1,0,1,0,1$ $0,0,1,0,1,0,1,0,0,1,0,1,1,0,0,0,0,1,1,1,1,0,0,0,0,1,1,0,0,0,1,0,0,0,1,0$ $0,0,1,0,0,1,1,1,0,0,1,0,0,1,0,0,1,0,1,1,1,0,0,0,0,1,0,0,0,0,0,1,0,1,0,1$ $0,0,1,0,0,1,0,0,1,1,1,0,0,1,0,0,1,0,1,0,0,1,1,0,1,0,1,0,1,0,0,0,1,0,0,0$ $0,0,0,1,1,1,1,0,0,1,0,0,0,0,1,0,1,0,1,0,0,0,0,1,0,0,1,1,0,1,0,0,1,0,1,0$ $0,0,0,1,1,0,0,1,1,0,1,1,0,0,0,1,1,0,0,1,0,0,1,0,0,1,0,0,0,0,1,1,0,0,1,0$ $0,0,0,1,1,0,0,1,1,0,0,0,1,1,0,0,0,1,1,1,0,0,1,0,1,0,1,1,0,1,0,0,0,0,0,0$ $0,0,0,1,0,1,1,1,0,0,0,0,1,1,0,1,0,1,0,0,1,1,0,0,1,0,0,0,1,0,0,1,1,0,0,0$ $0,0,0,1,0,1,0,0,1,1,1,1,0,0,0,1,0,1,0,0,1,1,0,0,0,1,1,1,0,1,0,0,0,0,0,0$

Figure 1: The matrix $A$.

1. $\Gamma$ is a Cayley graph for three different isomorphism types of groups.

2. Aut $(\Gamma)$ contains five conjugacy classes of regular subgroups, of which 4 are normal subgroups.

3. $\operatorname{Aut}(\Gamma)$ contains two isomorphic regular subgroups of shape $C_{3}^{2} \rtimes C_{2}^{2}$ for which one is normal in $\operatorname{Aut}(\Gamma)$ while the other is not, that is, $\Gamma$ is both a normal Cayley graph and a nonnormal Cayley graph for isomorphic groups.

Other examples of Cayley graphs that are both normal and nonnormal Cayley graphs for isomorphic groups are given in [1, 6].

In Example 1.2 we provide an infinite family of strongly regular graphs where we take $G$ to be a vertex-transitive proper subgroup of the full automorphism group and obtain normal quotients which are neither strongly regular nor complete.

Example 1.1. Let $\Gamma$ be the strongly regular graph with adjacency matrix $A$ given in Figure 1 which has parameters $\operatorname{srg}(36,14,4,6)$. The adjacency matrix was retrieved from [7]. 


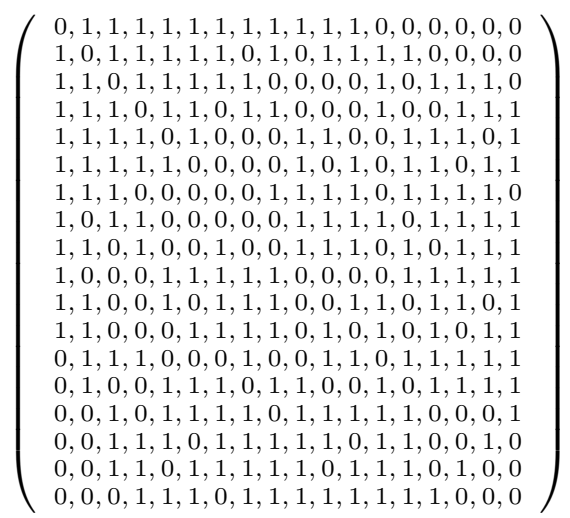

Figure 2: The matrix $B$.

According to a GAP [3] calculation, $\operatorname{Aut}(\Gamma)$ equals

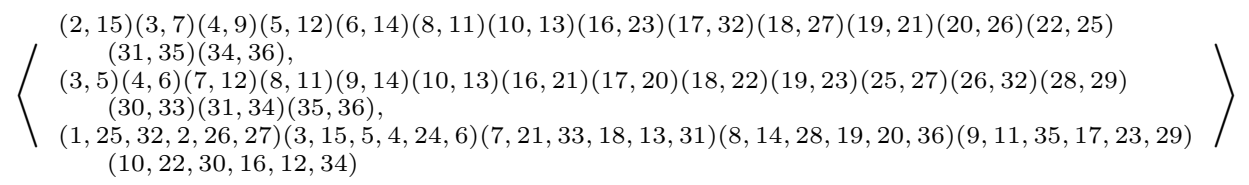

which has shape $C_{6}^{2} \rtimes C_{2}^{2}$. (By a group $G$ of shape $H \rtimes K$ we mean that $G$ has a normal subgroup $H$ and a subgroup $K$ such that $H \cap K=1$. Since this does not specify how $K$ acts on $H$ there may be more than one isomorphism class of groups of a given shape.) In fact, $G \cong \mathbb{Z}_{6}^{2} \rtimes\langle\sigma, \tau\rangle$ acting on $\mathbb{Z}_{6}^{2}$, with $\mathbb{Z}_{6}^{2}$ acting regularly on itself and $(a, b)^{\tau}=(-a,-b)$ and $(a, b)^{\sigma}=(b, a)$. Thus Aut $(\Gamma)$ is vertex-transitive and is a Cayley graph for $H_{1}=\mathbb{Z}_{6}^{2}$. The joining set is

$$
\begin{gathered}
\{(0,5),(0,1),(0,3),(5,0),(1,0),(3,0),(1,3),(5,3),(3,1),(3,5), \\
(1,5),(5,1),(2,4),(4,2)\} .
\end{gathered}
$$

Since $\operatorname{Aut}(\Gamma)_{(0,0)}=\langle\tau, \sigma\rangle$ has five orbits on this set, $\operatorname{Aut}(\Gamma)$ has five orbits on edges.

Now $\operatorname{Aut}(\Gamma)$ has a normal subgroup $N$ of order two generated by the element $(3,3) \in$ $\mathbb{Z}_{6}^{2}$ and which is the centre of $\operatorname{Aut}(\Gamma)$. The group $N$ has 18 orbits of length two on the 36 vertices of $\Gamma$ and the set of neighbours of $(0,0)$ contains the three $N$-orbits $\{(0,3),(3,0)\}$, $\{(1,5),(5,1)\}$ and $\{(2,4),(4,2)\}$. Hence, $\Gamma_{N}$ is a valency 11 graph on 18 vertices of diameter 2 but is not strongly regular. Indeed there are no feasible parameters for strongly regular graphs on 18 vertices which are not complete multipartite [4, p227]. The matrix $B$ given in Figure 2 is the adjacency matrix for $\Gamma_{N}$.

Not only is $\Gamma$ a Cayley graph for $H_{1}$, which is normal in $\operatorname{Aut}(\Gamma)$, we also have that $H_{2}=\langle(2,0),(0,2),(3,0) \tau,(0,3) \tau\rangle, H_{3}=\langle(0,2),(2,0),(3,0) \sigma\rangle$ and $H_{4}=\langle(2,0)$, $(0,2),(2,5) \sigma \tau\rangle$ are normal subgroups of $\operatorname{Aut}(\Gamma)$ that act regularly on $V \Gamma$. The subgroup $H_{2}$ has shape $C_{3}^{2} \rtimes C_{2}^{2}$, while $H_{3} \cong H_{4}$ have shape $C_{3}^{2} \rtimes C_{4}$. Finally, $H_{5}=$ $\langle(2,0),(0,2),(1,0) \tau,(0,1)\rangle \cong H_{2}$ is a regular subgroup of $\operatorname{Aut}(\Gamma)$ which is not normal. Thus $\Gamma$ is a Cayley graph for three different isomorphism types of groups. A Magma [2] 
calculation shows that $H_{1}, H_{2}, H_{3}, H_{4}$ and the subgroups conjugate to $H_{5}$ are the only regular subgroups of $\operatorname{Aut}(\Gamma)$.

The automorphism group of $\Gamma_{N}$ is isomorphic to $S_{2} \times S_{4} \times S_{3}$, which is vertex-transitive and has three orbits on edges. Note that $\operatorname{Aut}(\Gamma) / N<\operatorname{Aut}\left(\Gamma_{N}\right)$. The automorphism group contains 4 conjugacy classes of regular subgroups, none of which are normal in $\operatorname{Aut}\left(\Gamma_{N}\right)$. One class is isomorphic to $C_{3}^{2} \times C_{2}$, and there are three classes of subgroups with shape $C_{3}^{2} \rtimes C_{2}$, with two of the classes being isomorphic to each other. Representatives of these four conjugacy classes are $H_{i} / N$ for $i=1,2,3,4$. Note that $H_{2} / N=H_{5} N / N$.

Example 1.2. Let $\Gamma=H(2, m)$, the Hamming graph with $m^{2}$ vertices and suppose that $m$ is not a prime. Then $\Gamma$ is a strongly regular graph with parameters $\left(m^{2}, 2(m-1), m-2,2\right)$. Let $G=M_{1} \times M_{2}$, with $M_{1} \cong M_{2} \cong C_{m}$, act regularly on the set of vertices of $\Gamma$. Let $N_{1} \leqslant M_{1}$ and $N_{2} \leqslant M_{2}$ and $N=N_{1} \times N_{2} \triangleleft G$. Consider the graph $\Gamma_{N}$. Then $\Gamma_{N}$ is the cartesian product of $K_{r}$ and $K_{k}$ where $\left|M_{1}: N_{1}\right|=r$ and $\left|M_{2}: N_{2}\right|=k$. The adjacent vertices $\left(a, b_{1}\right),\left(a, b_{2}\right)$ in $\Gamma_{N}$ have $k-2$ common neighbours, namely the vertices of the form $(a, b)$ with $b \neq b_{1}, b_{2}$. However, the adjacent vertices $\left(a_{1}, b\right),\left(a_{2}, b\right)$ have $r-2$ common neighbours, these being the vertices of the form $(a, b)$ with $a \neq a_{1}, a_{2}$. Hence for $r \neq k$, the graph $\Gamma_{N}$ is not strongly regular.

\section{Acknowledgements}

The authors are grateful to the anonymous referees whose comments greatly improved the paper and led to Example 1.2.

\section{References}

[1] J. Bamberg and M. Giudici, Point regular automorphism groups of generalised quadrangles, submitted, arXiv:1005.2250v1.

[2] W. Bosma, J. Cannon and C. Playoust, The Magma algebra system, I. The user language. $J$. Symbolic Comput. 24 (1997) 235-265.

[3] The GAP Group, GAP-Groups, Algorithms, and Programming, Version 4.3, 2002, http: //www. gap-system.org.

[4] C. Godsil and G. F. Royle, Algebraic Graph Theory, Graduate Texts in Mathematics, 207, Springer-Verlag, New York, 2001.

[5] J. Morris, C. E. Praeger and P. Spiga, Strongly regular edge-transitive graphs, Ars Math. Contemp. 2 (2009), 137-155.

[6] G. F. Royle, A normal non-Cayley-invariant graph for the elementary abelian group of order 64, J. Aust. Math. Soc. 85 (2008), 347-351.

[7] E. Spence, http://www.maths.gla.ac.uk/ es/srgraphs.html. 